\title{
Editorial
}

\section{Does Dietary Pattern make any Effects on Depression?}

\author{
Yong Zhang \\ Department of Nutrition and Food Hygiene, School of Public Health and Health Management, Chongqing \\ Medical University, Chongqing, China \\ *Corresponding author: Yong Zhang, Department of Nutrition and Food Hygiene, School of Public \\ Health and Health Management, Chongqing Medical University, Chongqing, China, Tel: +86 $13594084999 ;$ \\ E-mail: zhangyongcq@live.cn
}

Received date: 27 March, 2018; Accepted date: 18 April, 2018; Published date: 25 April, 2018

Depression is a mental health problem with more than $10 \%$ prevalence [1] and the second leading cause of disability worldwide [2], accounted for $4.3 \%$ of global burden of disease alone [3]. It was estimated that depression would be the first leading cause of disability by 2030 [4], as Logan proposed that global urbanization and food industrialization might be its powerful promoters [5]. Therefore, it is extremely important to identify any modifiable risk factors that can help to develop effective prevention and control ways for depression. In this context, increasing interesting have been casted on population to demonstrate the association between nutrition and depression, from micro-level of nutrients, to macro-level of foods and dietary patterns during last decade.

It is almost clear that individual nutrients or foods such like n-3 PUFA, vitamin B group, fruits and vegetables on depression have limited effects to depression [6]. Considering the possible $\mathrm{cu}$ mulative or synergic effects of components presented in diet [7], many researchers believed dietary pattern rather than individual nutrient or food may play a more profound role in depression development and paid more attention to relationships between diet pattern and depression.

Generally, traditional diet, such as Mediterranean diet, Norwegian diet, Japanese diet, and DASH (Dietary Approaches to Stop Hypertension) diet designed for health purpose are identified as healthy diet, characterized by high intake of vegetable, fruits, whole grains, olive oil, fish, soy, poultry, and low fat dairy. Diets featured with high portion of red meat, processed meat, refined grains, sweets, high-fat or fried food, low-intake of fruits and vegetables on the contrary, are named as unhealthy diets or western-style diet.

However, studies in population often presented inconsistent results about the effects of diet on depression. As diet pattern is just one domain of multidimensional environmental exposures related to depression, to control most of the confounders at the same time is a big challenge. It is also difficult to collect foods consumption data accurately by questionnaire, which is the most common way for diet survey. Therefore, inherent methodological limitations in population studies may led to contradictive conclusions of the role playing by dietary pattern in depression.

Systematic reviews on dietary pattern and depression recently showed that healthy diet such as Mediterranean dietary pattern was positive and significant decrease the risk of depression, whereas unhealthy diet was negatively related to risk of depression [8]. However, some researchers argued that these conclusions mainly derived from cross-sectional studies which don't have the abilities to distinguish the causal relationships between two variables. In fact, people often changed their eating habit by taking more energy-rich food or sweetened-food when in low $\operatorname{mood}[9]$.

However, diet or nutrition has the self-evidenced impacts on brain and moods, because the brain has the highest energy and substances metabolic rate in order to maintain its structures and functions [10]. It is also worth mentioning that metabolic diseases and depression side by side have been becoming two epidemic diseases worldwide. This may indicate that both two types of diseases share the common etiologic mechanisms [11], or physiological diseases could induce psychological diseases by causing poor quality of life, limiting social function or reducing performance at work [12]. Studies have also found that healthy diet reduced the probability of depression more obviously in people with type 2 diabetes [13] and obesity [14]. Healthy diets, which were well testified to benefit diseases like obesity, hypertension, diabetes, cardiovascular disease, should benefit depression at the same time. In the future, more randomized controlled trails and prospective cohort studies are warranted to confirm the associations of diet patterns and depression. 
Even the high-grade evidences which directly support the benefits to depression are few in population level, we still can go a little further with precautions to provide some recommendations about dietary pattern [15] for public to control depression. The key elements of diet recommendation for depression control are including:

1. Follow 'traditional' and "prudent" diet, such as the Mediterranean, Norwegian, or Japanese traditional diet;

2. Follow DASH diet designed for hypertension and for overall health;

3. Follow plant-based diets which are rich in fruits, vegetables, legumes, wholegrain cereals, nuts, and seeds;

4. Choose diet with whole food instead of refined food;

5. Turn away from western-style diet with processed-foods, 'fast' foods, commercial bakery goods, and sweets.

\section{References}

1. Bromet E, Andrade LH, Hwang I, Sampson NA, Alonso J, et al. (2011) Cross-national epidemiology of DSM-IV major depressive episode. BMC Med 9: 90.

2. Ferrari AJ, Charlson FJ, Norman RE, Patten SB, Freedman G, et al. (2013) Burden of depressive disorders by country, sex, age, and year: findings from the global burden of disease study 2010. PLoS Med 10: e1001547.

3. Comprehensive mental health action plan 2013-2020. Mental Health, World Health Organization, Geneva.

4. Mathers C, Fat DM, Boerma JT (2008) The global burden of disease: 2004 update. World Health Organization.
5. Logan A C, Jacka F N (2014) Nutritional psychiatry research: an emerging discipline and its intersection with global urbanization, environmental challenges and the evolutionary mismatch. J Physiol Anthropol 33: 22.

6. Murakami K, Sasaki S (2010) Dietary intake and depressive symptoms: a systematic review of observational studies. Mol Nutr Food Res 54: 471-488.

7. Michels K B (2003) Nutritional epidemiology-past, present, future. Int J Epidemiology 32: 486-488.

8. Li Y, Lv MR, Wei Y J, Sun L, Zhang JX, et al. (2017) Dietary patterns and depression risk: a meta-analysis. Psychiatry Res 253: 373-382.

9. Camilleri G M, Méjean C, Kesse-Guyot E, Andreeva VA, Bellisle F, et al. (2014) The associations between emotional eating and consumption of energy-dense snack foods are modified by sex and depressive symptomatology. J Nutr 144: 1264-1273.

10. Beyer JL, Payne ME (2016) Nutrition and Bipolar Depression. Psychiatric Clinics of North America. 39: 75-86.

11. Murabito J M, Massaro J M, Clifford B, Udo Hoffmann, Caroline S Fox (2013) Depressive symptoms are associated with visceral adiposity in a community-based sample of middle-aged women and men. J Obesity 21: 1713-1719.

12. Lépine J P, Briley $M(2011)$ The increasing burden of depression. Neuropsychiatr Dis Treat 7: 3-7.

13. Dipnall J F, Pasco J A, Meyer D, Berk M, Williams LJ, et al. (2015) The association between dietary patterns, diabetes and depression. J J Affect Disord 174: 215-224.

14. Perezcornago A, Iglesia R D L, Lopezlegarrea P, Abete I, Navas-Carretero S, et al. (2014) A decline in inflammation is associated with less depressive symptoms after a dietary intervention in metabolic syndrome patients: a longitudinal study. J Nutrition Journal 13: 36.

15. Opie RS, Itsiopoulos C, Parletta N, Sanchez-Villegas A, Akbaraly TN, et al. (2015) Dietary recommendations for the prevention of depression. Nutr Neurosci 20: 161-171. 